\title{
TRIANGULARIZING SEMIGROUPS OF POSITIVE OPERATORS ON AN ATOMIC NORMED RIESZ SPACE
}

\author{
ROMAN DRNOVŠEK \\ Faculty of Mathematics and Physics, University of Ljubljana, Jadranska 19, \\ 1000 Ljubljana, Slovenia (roman.drnovsek@fmf.uni-lj.si)
}

(Received 20 August 1997)

\begin{abstract}
In the first part of the paper we prove several results on the existence of invariant closed ideals for semigroups of bounded operators on a normed Riesz space (of dimension greater than 1) possessing an atom. For instance, if $\mathcal{S}$ is a multiplicative semigroup of positive operators on such space that are locally quasinilpotent at the same atom, then $\mathcal{S}$ has a non-trivial invariant closed ideal. Furthermore, if $T$ is a non-zero positive operator that is quasinilpotent at an atom and if $\mathcal{S}$ is a multiplicative semigroup of positive operators such that $T S \leqslant S T$ for all $S \in S$, then $S$ and $T$ have a common non-trivial invariant closed ideal. We also give a simple example of a quasinilpotent compact positive operator on the Banach lattice $l^{\infty}$ with no non-trivial invariant band.

The second part is devoted to the triangularizability of collections of operators on an atomic normed Riesz space $L$. For a semigroup $S$ of quasinilpotent, order continuous, positive, bounded operators on $L$ we determine a chain of invariant closed bands. If, in addition, $L$ has order continuous norm, then this chain is maximal in the lattice of all closed subspaces of $L$.
\end{abstract}

Keywords: normed Riesz spaces; positive operators; invariant ideals; semigroups

AMS 1991 Mathematics subject classification: Primary 46B42; 47B65

\section{Introduction and preliminaries}

Invariant closed subspaces of bounded operators are among the most studied topics in the operator theory. In the case of positive operators on Banach lattices the order structure enable us to obtain invariant subspaces of simple geometrical forms. In [7] Choi et al. studied invariant closed subspaces of semigroups of positive operators on $L^{2}$-spaces. The discrete part of these results motivated our investigations. On the other hand, Abramovich et al. [2] obtained some results on existence of invariant closed subspaces of an operator on an $l^{p}$-space $(1 \leqslant p<\infty)$ which commutes with some non-zero locally quasinilpotent positive operator. In the present normed Riesz space setting, we are extending and strengthening the results of both papers. The proofs of our results mainly follow similar ideas that were introduced in $[2,3,7]$. It should be noted that Jahandideh $[\mathbf{8}, \mathbf{9}]$ also studied collections of positive operators. In [8] he considered the positive commutant of a given positive operator and collections of positive operators dominated 
by a given operator. In [9, Theorem 6] he proved a special case of our Theorem 2.4 , while in the rest of [9] operators on $A M$-spaces are studied.

The reader is assumed familiar with the general notions on normed Riesz spaces; see $[5,10,11,15,17]$. We shall recall some of the relevant facts. Let $L$ be a real normed Riesz space. The norm of $L$ is said to be order continuous if $\left\|f_{\tau}\right\| \downarrow 0$ for any downwards directed system $f_{\tau} \downarrow 0$ in $L\left(f_{\tau} \downarrow 0\right.$ means that $\inf \left\{f_{\tau}\right\}=0$, and for each $\tau_{1}$ and $\tau_{2}$ there exists $\tau_{3}$ such that $\left.f_{\tau_{3}} \leqslant \inf \left\{f_{\tau_{1}}, f_{\tau_{2}}\right\}\right)$. The space $L$ is Dedekind complete if every non-empty subset which is bounded from above has a supremum. The cone of positive elements of $L$ is denoted by $L^{+}$. The positive part, the negative part and the modulus (or the absolute value) of $f \in L$ are defined by $f^{+}=\sup \{f, 0\}, f^{-}=\sup \{-f, 0\}$ and $|f|=\sup \{f,-f\}$, respectively. A vector subspace $I$ of $L$ is said to be an (order) ideal if $|g| \leqslant|f|$ and $f \in I$ imply that $g \in I$. An ideal $I$ is called a band if $A \subseteq I$ with $\sup A \in L$ imply that $\sup A \in I$. Recall that bands are necessarily closed subspaces. Two elements $f, g \in L$ are called orthogonal or disjoint if $\inf \{|f|,|g|\}=0$. If $A$ is a subset of $L$, let $A^{d}$ be the disjoint complement of $A$, i.e. $f \in A^{d}$ if and only if $f$ is orthogonal to all elements of $A$. An atom of $L$ is a non-zero element $f \in L^{+}$such that if $0 \leqslant g \leqslant f$, then $g=\lambda f$ for some $\lambda \in[0,1]$. In other words, $f \in L^{+}$is an atom iff the ideal (or the band) generated by $f$ is one dimensional. In this case the ray $\mathcal{R}_{f}:=\{\lambda f: \lambda \geqslant 0\}$ is said to be an extreme ray. A normed Riesz space $L$ is said to be atomic if there exists an orthogonal maximal system of atoms $\left\{f_{\alpha}\right\}_{\alpha \in A} \subseteq L^{+}$, that is, $\inf \left\{f_{\alpha}, f_{\beta}\right\}=0$ if $\alpha \neq \beta$, and if inf $\left\{f, f_{\alpha}\right\}=0$ for all $\alpha \in A$, then $f=0$. It is worth mentioning that every atomic normed Riesz space is Riesz isomorphic to an order dense Riesz subspace of $\mathbb{R}^{A}$ with the coordinatewise order.

The word 'operator' will be synonymous with 'bounded linear transformation'. A subset $Z$ of $L$ is invariant under an operator $T$ on $L$ if $T f \in Z$ for all $f \in Z$. A subspace of $L$ is non-trivial if it is different from $\{0\}$ and $L$. If $\mathcal{C}$ is a collection of operators on $L$, then a subspace of $L$ is said to be $\mathcal{C}$-invariant whenever it is invariant under every member of $\mathcal{C}$. If this subspace is non-trivial, we also say that $\mathcal{C}$ has a non-trivial invariant subspace. An operator $T$ on $L$ is called positive if the positive cone $L^{+}$is invariant under $T$. A positive operator $T$ on $L$ is said to be order continuous if $T f_{\tau} \downarrow 0$ for any downwards directed system $f_{\tau} \downarrow 0$ in $L$. In general, the Banach algebra $\mathcal{B}(L)$ of all operators on $L$ is not a Riesz space under its canonical ordering ( $S \geqslant T$ iff the operator $S-T$ is positive). We therefore say that an operator $T$ on $L$ has a modulus if the modulus $|T|:=\sup \{T,-T\}$ exists in $\mathcal{B}(L)$.

If $P$ and $Q$ are any sets, we adopt the (standard) convention that $P \subset Q$ means $P \subseteq Q$ and $P \neq Q$. We first show the following result on the existence of closed ideals of a normed Riesz space.

Lemma 1.1. Let $I$ and $J$ be closed ideals of a normed Riesz space $L$ such that $I \subset J$ and the quotient space $G=J / I$ is at least two dimensional. Then there exists a closed ideal $K$ such that $I \subset K \subset J$.

Proof. Denote by $q$ the canonical (quotient) map $J \rightarrow G$. If we show that there exists a non-trivial closed ideal $\tilde{K}$ of $G$, then $K=q^{-1}(\tilde{K})$ is a closed ideal satisfying 
the condition of the lemma. Assume on the contrary that $\{0\}$ and $G$ are the only closed ideals of $G$. If $g \in G$, then $g^{+}=0$ or $g^{-}=0$. Indeed, if $g^{+} \neq 0$ and $g^{-} \neq 0$, then the closed ideal $I_{+} \neq\{0\}$ generated by $g^{+}$is proper, since $I_{+} \subseteq\left\{g^{-}\right\}^{d} \neq L$. Thus $g \geqslant 0$ or $g \leqslant 0$ for each $g \in G$, which imply that $G$ is totally ordered. By [15, Proposition II.3.4] $G$ is isomorphic to $\mathbb{R}$ and hence one dimensional. This contradiction completes the proof of the lemma.

A chain is a family of subspaces of $L$ that is totally ordered by inclusion. A collection $\mathcal{C}$ of operators on $L$ is ideal-triangularizable, if there is a chain of $\mathcal{C}$-invariant closed ideals which is maximal in the lattice of all closed ideals of $L$. This notion has been introduced in [8] as a Banach lattice analogue of the well-known concept of triangularizability (see, for example, [12]). Recall that a collection $\mathcal{C}$ of operators on $L$ is triangularizable, if there is a chain of $\mathcal{C}$-invariant closed subspaces which is maximal in the lattice of all closed subspaces of $L$. Using Lemma 1.1 we prove that a maximal closed ideal chain is also a maximal closed subspace chain.

Proposition 1.2. Let $\mathcal{C}$ be a chain of closed ideals of $L$ which is maximal in the lattice of all closed ideals. Then $\mathcal{C}$ is also maximal in the lattice of all closed subpaces of $L$.

Proof. By [14, Lemma 4.3.1] it is enough to show that the chain $\mathcal{C}$ is simple, i.e. it satisfies the following conditions:

(i) $\{0\} \in \mathcal{C}, L \in \mathcal{C}$;

(ii) if $\mathcal{C}_{0}$ is a subfamily of $\mathcal{C}$, then the closed subspaces

$$
\cap\left(I: I \in \mathcal{C}_{0}\right) \quad \text { and } \quad \operatorname{cl}\left(\cup\left(I: I \in \mathcal{C}_{0}\right)\right)
$$

are in $\mathcal{C}$, where cl denotes the norm closure;

(iii) for each $I \in \mathcal{C}$, the quotient space $I / I^{-}$, where

$$
I^{-}=\operatorname{cl}(\cup(J: J \in \mathcal{C}, J \subset I))
$$

is at most one dimensional. (We remark here that there is the standing hypothesis in [14] that spaces are complete. However, it is clear from the proof of [14, Lemma 4.3.1] that the completeness is not used.)

The condition (i) is clearly satisfied. Both subspaces of the condition (ii) are closed ideals. They therefore belong to $\mathcal{C}$, since $\mathcal{C}$ is maximal as a chain of closed ideals. The same fact and Lemma 1.1 imply easily that (iii) holds. This completes the proof.

The well-known theorem [13] states that a multiplicative semigroup $\mathcal{S}$ of operators on a Banach space has a non-trivial invariant closed subspace whenever a non-zero semigroup ideal of $\mathcal{S}$ has a non-trivial invariant closed subspace. (A subset $\mathcal{I}$ of a semigroup $\mathcal{S}$ is said to be a semigroup ideal if $S T$ and $T S$ belong to $\mathcal{I}$ for all $S \in \mathcal{S}$ and $T \in \mathcal{I}$.) The following is a normed Riesz space analogue of this very useful tool. It seems that this result (in the case of Banach lattices) appeared first in [9]. 
Lemma 1.3. Let $\mathcal{S}$ be a multiplicative semigroup of positive operators on a normed Riesz space $L$. If a non-zero semigroup ideal $\mathcal{I}$ of $\mathcal{S}$ has a non-trivial invariant closed ideal, then the semigroup $\mathcal{S}$ has a non-trivial invariant closed ideal as well.

Proof. Let $M$ be a non-trivial $\mathcal{I}$-invariant closed ideal. Let us show that the order ideal $N$ generated by the set

$$
\left\{T f: T \in \mathcal{I}, f \in M \cap L^{+}\right\}
$$

is $\mathcal{S}$-invariant. To end this, pick $g \in N$ and $S \in \mathcal{S}$. Since $g \in N$, there exist $\lambda \geqslant 0$, operators $T_{1}, T_{2}, \ldots, T_{n} \in \mathcal{I}$ and positive vectors $f_{1}, f_{2}, \ldots, f_{n} \in M$ such that

$$
|g| \leqslant \lambda\left(T_{1} f_{1}+\cdots+T_{n} f_{n}\right) .
$$

Then

$$
|S g| \leqslant S|g| \leqslant \lambda\left(S T_{1} f_{1}+\cdots+S T_{n} f_{n}\right) .
$$

Since $\mathcal{I}$ is a semigroup ideal, the operators $S T_{1}, \ldots, S T_{n}$ belong to $\mathcal{I}$, so that $S g \in N$. Thus the closure $\bar{N}$ of $N$ is a closed $\mathcal{S}$-invariant ideal. Since $N \subseteq M$, we have $\bar{N} \neq L$. So, if $N \neq\{0\}$, we are done. Assume therefore that $N=\{0\}$. Then the closed ideal

$$
K=\{f \in L: T|f|=0 \text { for all } T \in \mathcal{I}\}
$$

contains $M$. (Observe that $K$ is the intersection of all absolute kernels of operators of $\mathcal{I}$.) Since $\mathcal{I}$ is a non-zero ideal, $K$ is not equal to $L$, and so it is a non-trivial closed ideal. To show that $K$ is also $\mathcal{S}$-invariant, fix $f \in K$ and $S \in \mathcal{S}$. Then for any $T \in \mathcal{I}$ we have $0 \leqslant T|S f| \leqslant T S|f|=0$, since $T S \in \mathcal{I}$. This yields $S f \in K$, and completes the proof.

We now recall a notion from the local spectral theory that was first connected with the invariant subspace problems in [2]. An operator $T$ on $L$ is quasinilpotent at a vector $f_{0} \in L$ if the local spectral radius of $T$ at $f_{0}$ is 0 , i.e.

$$
\lim _{n \rightarrow \infty}\left\|T^{n} f_{0}\right\|^{1 / n}=0
$$

Throughout the rest of this section, let $L$ be an atomic normed Riesz space and $\left\{f_{\alpha}\right\}_{\alpha \in A} \subseteq L^{+}$the corresponding orthogonal maximal system of atoms. We may assume that $\left\|f_{\alpha}\right\|=1$ for all $\alpha \in A$. We now consider positive orthomorphisms on $L$, since they are needed in the last section of the paper. The first proposition is given in [15, Exercise II.7.] as an exercise. Recall that a family $\left\{g_{\tau}\right\}$ in $L$ order converges to $g \in L$ whenever there exists a downwards directed system $p_{\tau} \downarrow 0$ in $L$ such that $\left|g_{\tau}-g\right| \leqslant p_{\tau}$ for all $\tau$.

Proposition 1.4. Every $f \in L$ has a unique representation

$$
f=\sum_{\alpha \in A} \lambda_{\alpha} f_{\alpha}, \quad \lambda_{\alpha} \in \mathbb{R},
$$

where the sum is the order limit of its finite subsums. 
Sketch of proof. Fix $f \in L^{+}$and set $\lambda_{\alpha}=\sup \left\{\lambda \geqslant 0: \lambda f_{\alpha} \leqslant f\right\}$ for each $\alpha \in A$. Since $L$ is Archimedean, $\lambda_{\alpha} f_{\alpha} \leqslant f$. If $\mathcal{A}$ denotes the collection of all finite subsets of $A$, then the inequality $g_{B}=\sum_{\alpha \in B} \lambda_{\alpha} f_{\alpha} \leqslant f$ holds for each $B \in \mathcal{A}$. For the proof of this, use the equality $\sup \{u, v\}=u+v$, where $u, v \in L$ and $\inf \{u, v\}=0$. It is easily seen that $f$ is the supremum of the upwards directed system $\left\{g_{B}\right\}_{B \in \mathcal{A}}$, that is, each $f \in L^{+}$ has the desired representation. This is also true for any $f \in L$, since $f=f^{+}-f^{-}$. The uniqueness of the representation follows from the fact that $\left\{f_{\alpha}\right\}_{\alpha \in A}$ are orthogonal.

Observe now that for any $\alpha \in A$ the positive linear functional $\phi_{\alpha}$ on $L$ with norm 1 is defined by $\phi_{\alpha}(f)=\lambda_{\alpha}$.

An operator on $L$ is called an orthomorphism $[\mathbf{1 7}$, p. 648$]$ if it leaves invariant every band in $L$. It follows from [17, Lemma 144.1] that a positive operator $D$ on $L$ is an orthomorphism if and only if there exists a $\lambda>0$ such that $D \leqslant \lambda I$, where $I$ is the identity operator on $L$. This implies that a positive orthomorphism on $L$ leaves invariant every ideal in $L$.

Proposition 1.5. Let $L$ be Dedekind complete, and let $T$ be a positive operator on $L$. Then for each $f \in L$ the order limit of finite subsums of the sum

$$
D f=\sum_{\alpha \in A} \phi_{\alpha}\left(T f_{\alpha}\right) \phi_{\alpha}(f) f_{\alpha}
$$

exists in $L$, and $D$ is a positive orthomorphism on $L$. If, in addition, $T$ is an orthomorphism, then $D=T$.

Proof. We may assume that $f \in L^{+}$. Since $\phi_{\alpha}\left(T f_{\alpha}\right) \leqslant\|T\|$ for each $\alpha \in A$, we conclude that each finite subsum of $D f$ is less than or equal to $\|T\| f$. This implies first that $D f$ exists, since $L$ is Dedekind complete, and $D f \leqslant\|T\| f$, so that $D$ is a positive orthomorphism.

Assume that $T$ is an orthomorphism. Since there is a $\lambda>0$ such that $T f_{\alpha} \leqslant \lambda f_{\alpha}$ for all $\alpha \in A$, we conclude that $T f_{\alpha}=\phi_{\alpha}\left(T f_{\alpha}\right) f_{\alpha}$. Hence $T f_{\alpha}=D f_{\alpha}$ for all $\alpha \in A$. Consequently, $D=T$ by [17, Corollary 140.6.(ii)].

Proposition 1.6. Let $L$ be Dedekind complete, and let $T$ be a positive operator on $L$. Then $T$ has a unique decomposition $T=D_{T}+N_{T}$, where $D_{T}$ is a positive orthomorphism on $L$ and $N_{T}$ is a positive operator satisfying $\phi_{\alpha}\left(N_{T} f_{\alpha}\right)=0$ for all $\alpha \in A$. Moreover, the operators $D_{T}$ and $N_{T}$ are given by

$$
D_{T} f=\sum_{\alpha \in A} \phi_{\alpha}\left(T f_{\alpha}\right) \phi_{\alpha}(f) f_{\alpha} \quad \text { and } \quad N_{T} f=\sum_{\alpha \in A} \phi_{\alpha}\left(T\left(f-\phi_{\alpha}(f) f_{\alpha}\right)\right) f_{\alpha}
$$

for each $f \in L$.

Proof. The operator $D_{T}$ defined by (1.2) is a positive orthomorphism on $L$ by Proposition 1.5. Setting $N_{T}=T-D_{T}$ the equality $T f=\sum_{\alpha \in A} \phi_{\alpha}(T f) f_{\alpha}$ gives the second 
equality of (1.2). The operator $N_{T}$ is positive, because $f \geqslant \phi_{\alpha}(f) f_{\alpha}$ for all $f \in L^{+}$and for all $\alpha \in A$. Also, $\phi_{\alpha}\left(N_{T} f_{\alpha}\right)=0$ for all $\alpha \in A$. We have therefore proved that $T$ has the desired decomposition. To show its uniqueness, assume that $T=D_{1}+N_{1}$ is another such decomposition. Since $\phi_{\alpha}\left(D_{1} f_{\alpha}\right)=\phi_{\alpha}\left(T f_{\alpha}\right)$ for each $\alpha \in A$, we conclude that $D_{T}=D_{1}$ by putting $D:=D_{T}$ and $T:=D_{1}$ in Proposition 1.5.

If $T$ is a positive operator on $L$ satisfying $\phi_{\alpha}\left(T f_{\alpha}\right)=0$ for all $\alpha \in A$, then it is obvious that $D_{T}=0$ and $N_{T}=T$. Of course, in this case we do not need the assumption that $L$ is Dedekind complete.

Although orthomorphisms and the above decomposition of operators on an arbitrary Riesz space have been extensively studied (see [17] or [5]), we include here an example for those readers that are not familiar with this topic.

Example 1.7. Let $L=l^{p}, 1 \leqslant p \leqslant \infty$. Then the sequence of standard unit vectors $\left\{e_{n}\right\}_{n \in \mathbb{N}}$ is the corresponding orthogonal maximal system of atoms. Each positive operator $T$ on $l^{p}$ can be represented by a matrix $\left[t_{i j}\right]_{i, j=1}^{\infty}$, where non-negative numbers $t_{i j}$ satisfy equations

$$
T e_{j}=\sum_{i=1}^{\infty} t_{i j} e_{i}, \quad j \in \mathbb{N} .
$$

Here the sum is taken as an order limit of its finite subsums. It is clear that $D_{T}$ is the 'diagonal part' of $T$, that is, the corresponding matrix of $D_{T}$ is a diagonal matrix with the sequence $\left\{t_{i i}\right\}_{i \in \mathbb{N}}$ on the diagonal. The operator $N_{T}$ is the 'non-diagonal part' of $T$, that is, its matrix has zero diagonal, while non-diagonal elements are the same as in the matrix of $T$.

\section{A common invariant closed ideal}

Let $L$ be a real normed Riesz space of dimension greater than 1 , and let $f_{0} \in L^{+}$be an atom. The band generated by $f_{0}$ in $L$ is denoted by $B_{0}$. One can show that $B_{0}$ is a projective band of $L$, i.e. $L=B_{0} \oplus B_{0}^{d}$ (see [10, Theorem 26.4, p. 147]). Therefore, for each $f \in L$ there exist (uniquely determined) $\lambda \in \mathbb{R}$ and $g \in B_{0}^{d}$ such that $f=\lambda f_{0}+g$. Let $\phi_{0}$ be the positive linear functional on $L$ defined by $\phi_{0}(f)=\lambda$. Since the vectors $\lambda f_{0}$ and $g$ are orthogonal, $|f|=|\lambda| f_{0}+|g|$. Then $|\lambda| f_{0} \leqslant|f|$ implies that $\left\|\phi_{0}\right\| \leqslant 1$. Since $\phi_{0}\left(f_{0}\right)=1$, we have $\left\|\phi_{0}\right\|=1$.

Theorem 2.1. Let $f_{0} \in L^{+}$be an atom and let $\mathcal{S}$ be a multiplicative semigroup of positive operators on $L$ such that $\phi_{0}\left(S f_{0}\right)=0$ for all $S \in \mathcal{S}$. Then $\mathcal{S}$ has a non-trivial invariant closed ideal. If, in addition, each member of $\mathcal{S}$ is order continuous, then $\mathcal{S}$ has a non-trivial invariant closed band.

Proof. If $S f_{0}=0$ for all $S \in \mathcal{S}$, then $B_{0}$ is a non-trivial $\mathcal{S}$-invariant band. Therefore, we have to consider the case when $S f_{0} \neq 0$ for some $S \in \mathcal{S}$. 
Let $I$ be the order ideal generated in $L$ by the set $\left\{S f_{0}: S \in S\right\}$, i.e. $I$ is the ideal of all $f \in L$ such that there exist $\lambda \geqslant 0$ and $S_{1}, S_{2}, \ldots, S_{n} \in \mathcal{S}$ such that $|f| \leqslant \lambda\left(S_{1}+S_{2}+\cdots+S_{n}\right) f_{0}$. We claim that $I$ is invariant under arbitrary $S \in \mathcal{S}$. To show this, pick $f \in I$. Then $|f| \leqslant \lambda\left(S_{1}+S_{2}+\cdots+S_{n}\right) f_{0}$ for some $\lambda \geqslant 0$ and $S_{1}, S_{2}$, $\ldots, S_{n} \in \mathcal{S}$, and hence

$$
|S f| \leqslant S|f| \leqslant \lambda\left(S S_{1}+S S_{2}+\cdots+S S_{n}\right) f_{0} .
$$

Since $\mathcal{S}$ is a semigroup, we conclude that $S f \in I$. Now, the closure $\bar{I}$ of $I$ is invariant under $S$ as well, because the operator $S$ is continuous. Since $\phi_{0}\left(S f_{0}\right)=0$, we have $S f_{0} \in B_{0}^{d}$ for all $S \in \mathcal{S}$, so that $\{0\} \neq \bar{I} \subseteq B_{0}^{d} \neq L$. Thus we have shown that $\bar{I}$ is a non-trivial $\mathcal{S}$-invariant closed ideal, and so the proof of the first assertion is finished.

Now assume that each member of $\mathcal{S}$ is order continuous. Then the band $B$ generated by $I$ is non-trivial, since $B \subseteq B_{0}^{d} \neq L$. It is also invariant under each $S \in \mathcal{S}$. Indeed, for each $f \in B \cap L^{+}$there exists an upwards directed system $\left\{f_{\tau}\right\}$ of vectors in $I$ such that $0 \leqslant f_{\tau} \uparrow f$. Hence $0 \leqslant S f_{\tau} \uparrow S f$, since $S$ is order continuous. This implies that $S f \in B$.

As a consequence we obtain the following theorem.

Theorem 2.2. Let $f_{0} \in L^{+}$be an atom and let $\mathcal{S}$ be a multiplicative semigroup of positive operators on $L$ such that each of them is quasinilpotent at $f_{0}$. Then $\mathcal{S}$ has a non-trivial invariant closed ideal. If, in addition, each member of $\mathcal{S}$ is order continuous, then there exists a non-trivial $\mathcal{S}$-invariant band.

Proof. Fix $S \in \mathcal{S}$ and set $\lambda=\phi_{0}\left(S f_{0}\right)$. From $S f_{0} \geqslant \lambda f_{0}$ we conclude that $S^{n} f_{0} \geqslant$ $\lambda^{n} f_{0}$ for all $n \in \mathbb{N}$, so that $\lambda\left\|f_{0}\right\|^{1 / n} \leqslant\left\|S^{n} f_{0}\right\|^{1 / n}$. Since $S$ is quasinilpotent at $f_{0}$, this gives $\lambda=0$, and so the condition of Theorem 2.1 is satisfied.

One may ask if we can drop the additional assumption of Theorems 2.1 and 2.2 that each member of $\mathcal{S}$ is order continuous. In the next example we construct a quasinilpotent compact positive operator $S$ on $l^{\infty}$ with no non-trivial invariant band. Consequently, the semigroup $\mathcal{S}$ generated by $S$ does not have a non-trivial invariant band.

Example 2.3. Let $\phi$ be a Banach limit on $l^{\infty}$. (Actually, it is enough that $\phi$ is a positive linear functional on $l^{\infty}$ such that $\phi(x)=\lim _{n \rightarrow \infty} x_{n}$ for each $x=\left(x_{1}, x_{2}, x_{3}, \ldots\right)$ from the Banach subspace $c$ of all convergent sequences of $l^{\infty}$.) Let

$$
u=(1,1 / 2,1 / 4,1 / 8, \ldots) \in l^{\infty} .
$$

Define the compact positive operators $K$ and $T$ on $l^{\infty}$ by

$$
K x=\phi(x) u \quad \text { and } T x=\left(0, x_{1} / 2, x_{2} / 4, x_{3} / 8, \ldots\right) .
$$

Obviously, the operator $K$ is nilpotent of index 2 . Since $\left\|T^{n}\right\|=2^{-n(n+1) / 2}$ for all $n \in \mathbb{N}$, the operator $T$ is a quasinilpotent. Clearly, $S=K+T$ is a compact positive operator 
on $l^{\infty}$. Since $K^{2}=0$ and $K T=0$, we have $K S=0$. By a simple induction we obtain that $S^{n}=T^{n-1} S$, and so $\left\|S^{n}\right\| \leqslant\left\|T^{n-1}\right\|\|S\|$ for all $n \in \mathbb{N}$. It follows from this that the operator $S$ is quasinilpotent as well.

We now show that $S$ has no non-trivial invariant band. Assume that a band $B \neq\{0\}$ is invariant under $S$. Then $e_{n} \in B$ for some $n \in \mathbb{N}$, where $\left\{e_{i}\right\}_{i \in \mathbb{N}}$ are the standard unit vectors of $l^{\infty}$. From $S e_{n+k}=T e_{n+k}=\left(1 / 2^{n+k}\right) e_{n+k+1}, k=0,1,2,3, \ldots$, we conclude that $e_{n+k} \in B$ for all $k \in \mathbb{N}$. It follows that $g=\sum_{k=0}^{\infty} e_{n+k} \in B$, because $g$ is the supremum of the increasing sequence $\left\{g_{m}\right\}_{m \in \mathbb{N}}$ of $B$ defined by $g_{m}=\sum_{k=0}^{m} e_{n+k}$. Since $S g \geqslant K g=u$, we have $u \in B$ (as $B$ is an ideal), and so $B=l^{\infty}$.

In the study [1] of various extensions of de Pagter's and the Ando-Krieger results Abramovich et al. constructed an example of quasinilpotent compact positive operator with no non-trivial invariant band. This operator is defined on a less-known Marcinkiewicz function space. Therefore, the operator $S$ of Example 2.3 seems to be a new example of such operators, which is in addition defined on a nice atomic Banach lattice. However, it should be noted that the operator of the example in [1] is even a nilpotent rank-one operator.

Theorem 2.4. Let $T$ be a non-zero positive operator on $L$ that is quasinilpotent at an atom $f_{0} \in L^{+}$. Let $\mathcal{S}$ be a multiplicative semigroup of operators such that each of them has a modulus. If $T|S| \leqslant|S| T$ for all $S \in \mathcal{S}$, then $T$ has a non-trivial invariant closed ideal that is also $\mathcal{S}$-invariant.

Proof. Let $\mathcal{T}$ be a semigroup generated by $\mathcal{T}$ and the set $\{|S|: S \in \mathcal{S}\}$. Denote by $\mathcal{I}$ the semigroup ideal of $\mathcal{T}$ generated by $T$. We claim that each member of $\mathcal{I}$ is quasinilpotent at $f_{0}$. Indeed, if $U \in \mathcal{I}$, then $U \leqslant\left|S_{1}\right|\left|S_{2}\right| \ldots\left|S_{p}\right| T^{k}$ for some $k \in \mathbb{N}$, $p \in \mathbb{N} \cup\{0\}$, and $S_{1}, \ldots, S_{p} \in \mathcal{S}$. Denoting $V=\left|S_{1}\right|\left|S_{2}\right| \ldots\left|S_{p}\right| T^{k-1}$ we have $U \leqslant V T$. Since $T V \leqslant V T$ by the assumption on $\mathcal{S}$, an easy induction shows that $U^{n} \leqslant V^{n} T^{n}$ for all $n \in \mathbb{N}$. We therefore have

$$
\left\|U^{n} f_{0}\right\| \leqslant\left\|V^{n} T^{n} f_{0}\right\| \leqslant\|V\|^{n} \cdot\left\|T^{n} f_{0}\right\|
$$

for all $n$, which implies that $U$ is quasinilpotent at $f_{0}$. By Theorem 2.2 the semigroup ideal $\mathcal{I}$ has a non-trivial invariant closed ideal. Now Lemma 1.3 gives a non-trivial $\mathcal{T}$ invariant closed ideal $I$. Let us show that $I$ is $\mathcal{S}$-invariant as well. To end this, let $f \in I$ and $S \in \mathcal{S}$. Since $|f| \in I$ and $|S| \in \mathcal{T}$, we conclude that $|S||f| \in I$, and finally the inequality $|S f| \leqslant|S||f|$ implies that $S f \in I$ (as $I$ is an order ideal).

Corollary 2.5. Let $S$ be an operator on $L$ with modulus and let $T$ be a non-zero positive operator on $L$ such that

(i) $T|S| \leqslant|S| T$, and

(ii) $T$ is quasinilpotent at an atom of $L^{+}$,

then $S$ and $T$ have a common non-trivial invariant closed ideal. 
One can show easily that if a positive operator on an atomic Riesz normed space is quasinilpotent at a non-zero positive vector, then it is quasinilpotent at some atom as well. This observation together with Corollary 2.5 gives the following.

Corollary 2.6. Let $S$ be an operator with modulus on an atomic Riesz normed space $L$. If there exists a non-zero positive operator $T$ on $L$ such that

(i) $T|S| \leqslant|S| T$, and

(ii) $T$ is quasinilpotent at a non-zero positive vector,

then $S$ and $T$ have a common non-trivial invariant closed ideal.

Using the fact that every closed ideal of a Banach lattice with order continuous norm is a band (see, for example, [17, Theorem 105.2]), the main result of [2] follows from Corollary 2.6. In fact, we state here a slight improvement of [2, Theorem 3.1], which has been proved in the survey paper [4].

Corollary 2.7. [2,4] Let $L$ be an atomic Banach lattice with order continuous norm, and let $S$ be an operator on $L$ with modulus. If there exists a non-zero positive operator $T$ on $L$ such that

(i) $T|S| \leqslant|S| T$, and

(ii) $T$ is quasinilpotent at a non-zero positive vector,

then $S$ has a non-trivial invariant band.

We shall prove next that the conclusion of Theorem 2.2 holds also for additive semigroups of positive operators. In the proof of this we need the following result.

Proposition 2.8. Let $\mathcal{S}$ be an additive semigroup of positive operators on $L$ such that each of them is quasinilpotent at an atom $f_{0} \in L^{+}$. Then every operator in the algebra generated by $\mathcal{S}$ is quasinilpotent at $f_{0}$.

Proof. Let $A$ be an operator in the algebra generated by $\mathcal{S}$. Then there exist positive integers $m$ and $p$ and operators $S_{1}, \ldots, S_{k} \in \mathcal{S}$ such that $A=\sum_{i=1}^{m} a_{i} A_{i}$, where $a_{i} \in \mathbb{R}$ and each $A_{i}$ is a product of at most $p$ (not necessarily distinct) elements of $\left\{S_{1}, \ldots, S_{k}\right\}$. Let $S=S_{1}+\cdots+S_{k}$ and $T=\sum_{j=0}^{p-1} S^{j}$. From $\left\|(S T)^{n} f_{0}\right\| \leqslant\|T\|^{n}\left\|S^{n} f_{0}\right\|$ it follows that $S T$ is quasinilpotent at $f_{0}$. This implies that the operator $\tilde{A}=\sum_{i=1}^{m}\left|a_{i}\right| A_{i}$ is also quasinilpotent at $f_{0}$, since $\tilde{A} \leqslant a \sum_{j=1}^{p} S^{j} \leqslant a S T$, where $a=\max _{1 \leqslant i \leqslant m}\left\{\left|a_{i}\right|\right\}$. From the fact that $\left|A_{i} f\right| \leqslant A_{i}|f|$ for all $f \in L$ it follows easily that $|A f| \leqslant \tilde{A}|f|$ for all $f \in L$. Hence $\left|A^{n} f_{0}\right| \leqslant \tilde{A}^{n} f_{0}$, and so $\left\|A^{n} f_{0}\right\| \leqslant\left\|\tilde{A}^{n} f_{0}\right\|$ for all $n \in \mathbb{N}$. From this it follows that $A$ is quasinilpotent at $f_{0}$ as well.

Theorem 2.9. Let $\mathcal{S}$ be an additive semigroup of positive operators on $L$ that are quasinilpotent at an atom $f_{0} \in L^{+}$. Then there exists a non-trivial $\mathcal{S}$-invariant closed ideal $I$. If each member of $\mathcal{S}$ is order continuous, then $I$ can be chosen to be a band. 
Proof. Proposition 2.8 implies that all operators of the multiplicative semigroup $\mathcal{T}$ generated by $\mathcal{S}$ are quasinilpotent at $f_{0}$. Now apply Theorem 2.2 for the semigroup $\mathcal{T}$. For the proof of the second assertion, observe first that a product of order continuous operators is order continuous. Hence every operator of $\mathcal{T}$ is order continuous, and the second assertion of Theorem 2.2 applies.

\section{A maximal chain of common invariant bands}

We first answer negatively the question posed in [8] whether every quasinilpotent positive operator on a Banach lattice, which contains an atom, is ideal-triangularizable.

Example 3.1. Let $E=L^{p}(\Gamma, \mu)(1 \leqslant p<\infty)$, where $\mu$ is a Haar measure on the circle group $\Gamma$. Schaefer [16] (see also [11, §4.2]) constructed a quasinilpotent positive operator $T$ on $E$ with no non-trivial invariant closed ideal. Let $L$ be the direct sum of Banach lattices $E$ and $\mathbb{R}$. Then $L$ is a Banach lattice with an atom $0 \oplus 1$, and the operator $S=T \oplus 0$ is a quasinilpotent positive operator on $L$ whose only non-trivial invariant closed ideals are $E \oplus 0$ and $0 \oplus \mathbb{R}$. Therefore, $S$ is not ideal-triangularizable.

From now on, let $L$ be an atomic normed Riesz space of dimension greater than 1 , and let $\left\{f_{\alpha}\right\}_{\alpha \in A} \subseteq L^{+}$be the corresponding orthogonal maximal system of atoms.

Theorem 3.2. Let $L$ be Dedekind complete, and let $\mathcal{S}$ be a collection of positive operators on $L$ with the following property: whenever there is an $S \in \mathcal{S}$ such that $\phi_{\beta}\left(N_{S} f_{\alpha}\right)>0$ and a $T \in \mathcal{S}$ such that $\phi_{\gamma}\left(N_{T} f_{\beta}\right)>0$, then there is a $U \in \mathcal{S}$ such that $\phi_{\gamma}\left(N_{U} f_{\alpha}\right)>0$. Then there is a total ordering of the index set $A$ such that $\phi_{\beta}\left(N_{S} f_{\alpha}\right)=0$ whenever $S \in \mathcal{S}$ and $\alpha \leqslant \beta$.

Proof. Define an order on $A$ by : $\alpha>\beta$ if there exists an operator $S \in \mathcal{S}$ such that $\phi_{\beta}\left(N_{S} f_{\alpha}\right)>0$. Set $\alpha \geqslant \beta$ if either $\alpha>\beta$ or $\alpha=\beta$. We claim that $\geqslant$ is a partial ordering. If $\alpha>\beta$ and $\beta>\alpha$, then there are $S, T \in \mathcal{S}$ such that $\phi_{\beta}\left(N_{S} f_{\alpha}\right)>0$ and $\phi_{\alpha}\left(N_{T} f_{\beta}\right)>0$. Then, by our assumption, there exists a $U \in \mathcal{S}$ such that $\phi_{\alpha}\left(N_{U} f_{\alpha}\right)>0$ which is impossible. This proves that $\geqslant$ is antisymmetric. The same assumption also implies that $\geqslant$ is transitive. Using Zorn's lemma one can show that the partial ordering $\geqslant$ can be extended to a total ordering that we denote by $\geqslant$ as well. Now, if $\beta>\alpha$ and $\phi_{\beta}\left(N_{S} f_{\alpha}\right)>0$ for some $S \in \mathcal{S}$, then $\alpha>\beta$, and hence $\alpha>\alpha$ which is a contradiction.

Let $\geqslant$ be a total ordering of the index set $A$, obtained in Theorem 3.2. Let $\mathcal{D}(A)$ be the Dedekind completion (by cuts) of the set $A$ (see, for example, [6]). We adjoin a smallest element and a largest element to $\mathcal{D}(A)$, unless $\mathcal{D}(A)$ has these elements already. Let $\mathcal{H}$ be the collection of all subsets of $A$ that have the form either $\{\alpha \in A: \alpha<\delta\}$ or $\{\alpha \in A: \alpha \leqslant \delta\}$ for some $\delta \in \mathcal{D}(A)$. Observe that $\mathcal{H}$ is closed under arbitrary unions and intersections, and $\emptyset \in \mathcal{H}, A \in \mathcal{H}$. For any $H \in \mathcal{H}$ let $B_{H}$ be the band generated in $L$ by the set $\left\{f_{\alpha}: \alpha \in H\right\}$. (If $H=\emptyset$, then $B_{H}=\{0\}$.) Observe that $B_{H}=\mathrm{\cap}\left(\operatorname{Ker}\left(\phi_{\alpha}\right): \alpha \notin H\right)=\cap\left(\left\{f_{\alpha}\right\}^{d}: \alpha \notin H\right)$. Denote $\mathcal{C}=\left\{B_{H}: H \in \mathcal{H}\right\}$. 
Theorem 3.3. Assume that all members of the collection $\mathcal{S}$ of Theorem 3.2 are order continuous. Then the chain $\mathcal{C}$ consists of $\mathcal{S}$-invariant bands, and it is maximal in the lattice of all bands of $L$. If, in addition, $L$ has order continuous norm, then the chain $\mathcal{C}$ is even maximal in the lattice of all closed subspaces of $L$.

Proof. Pick $H \in \mathcal{H}, \alpha \in H$ and $S \in \mathcal{S}$. Since $\phi_{\beta}\left(N_{S} f_{\alpha}\right)=0$ for all $\beta \geqslant \alpha$, we have

$$
N_{S} f_{\alpha}=\sum_{\beta \in A} \phi_{\beta}\left(N_{S} f_{\alpha}\right) f_{\beta}=\sum_{\beta<\alpha} \phi_{\beta}\left(N_{S} f_{\alpha}\right) f_{\beta}
$$

so that $N_{S} f_{\alpha} \in B_{H}$ as $B_{H}$ is a band. Because $D_{S} f_{\alpha} \in B_{H}$ as well, we obtain that $S f_{\alpha}=D_{S} f_{\alpha}+N_{S} f_{\alpha} \in B_{H}$. Since each $f \in B_{H}$ is an order limit of elements belonging to the linear hull of $\left\{f_{\alpha}: \alpha \in H\right\}$, since $S$ is order continuous and since $B_{H}$ is a band, we conclude easily that $B_{H}$ is invariant under $S$. We have therefore shown that $\mathcal{C}$ consists of $\mathcal{S}$-invariant bands.

Assume now that $\mathcal{C}$ is not maximal in the lattice of all bands. Then there exists a band $B \notin \mathcal{C}$ such that $\mathcal{C} \cup\{B\}$ is still a chain of bands. Let $B_{1}$ be the supremum of all bands of $\mathcal{C}$ that are contained in $B$, and let $B_{2}$ be the infimum (intersection) of all bands of $\mathcal{C}$ that contain $B$. Since $\mathcal{D}(A)$ is Dedekind complete, the bands $B_{1}$ and $B_{2}$ belong to $\mathcal{C}$. From $B_{1} \subset B \subset B_{2}$ we conclude that there exists $\alpha \in A$ such that $f_{\alpha} \in B \backslash B_{1}$. Let $H=\{\beta \in A: \beta \leqslant \alpha\}$. Then the band $B_{H}$ satisfies inclusions $B_{1} \subset B_{H} \subset B_{2}$. Since $\mathcal{C} \cup\{B\}$ is a chain of bands, we have either $B_{1} \subset B_{H} \subset B$ or $B \subset B_{H} \subset B_{2}$. In either case we obtain a contradiction with the definitions of the bands $B_{1}$ and $B_{2}$. Thus $\mathcal{C}$ is maximal in the lattice of all bands of $L$.

If the norm of $L$ is order continuous, then each closed ideal is a band (see, for example, [17, Theorem 105.2]). Thus, the chain $\mathcal{C}$ is maximal in the lattice of all closed ideals of $L$, and so, by Proposition 1.2, the chain $\mathcal{C}$ is also maximal in the lattice of all closed subspaces of $L$.

Corollary 3.4. Let $L$ be Dedekind complete, and let $\mathcal{S}$ be a multiplicative semigroup of order continuous positive operators on $L$. Assume that $\phi_{\alpha}\left(N_{S} N_{T} f_{\alpha}\right)=0$ for all $\alpha \in A$ and for all $S, T \in \mathcal{S}$. Then for $\mathcal{S}$ the conclusions of Theorems 3.2 and 3.3 hold.

Proof. Let us show first that for any $S, T \in \mathcal{S}$ we have $D_{S T}=D_{S} D_{T}$ and $N_{S T}=$ $D_{S} N_{T}+N_{S} D_{T}+N_{S} N_{T}$. In view of the characterization of positive orthomorphisms preceding Proposition 1.5, there exist positive numbers $\lambda$ and $\mu$ such that $D_{S} \leqslant \lambda I$ and $D_{T} \leqslant \mu I$, where $I$ denotes the identity operator on $L$. It follows that $D_{S} D_{T} \leqslant \lambda \mu I$, so that the operator $D_{S} D_{T}$ is an orthomorphism. Denoting $N:=D_{S} N_{T}+N_{S} D_{T}+N_{S} N_{T}$ we have, for any $\alpha \in A$,

$$
0 \leqslant \phi_{\alpha}\left(N f_{\alpha}\right) \leqslant \lambda \phi_{\alpha}\left(N_{T} f_{\alpha}\right)+\mu \phi_{\alpha}\left(N_{S} f_{\alpha}\right)+\phi_{\alpha}\left(N_{S} N_{T} f_{\alpha}\right)=0,
$$

which yields $\phi_{\alpha}\left(N f_{\alpha}\right)=0$. Since $S T=D_{S} D_{T}+N$, the uniqueness of the decomposition implies that $D_{S T}=D_{S} D_{T}$ and $N_{S T}=N$. 
We now show that $\mathcal{S}$ satisfies the assumption of Theorem 3.2. Suppose that $a=$ $\phi_{\beta}\left(N_{S} f_{\alpha}\right)>0$ and $b=\phi_{\gamma}\left(N_{T} f_{\beta}\right)>0$, so that $N_{S} f_{\alpha} \geqslant a f_{\beta}$ and $N_{T} f_{\beta} \geqslant b f_{\gamma}$. Then $N_{S T} f_{\alpha} \geqslant N_{S} N_{T} f_{\alpha} \geqslant a b f_{\gamma}$, and hence $\phi_{\gamma}\left(N_{S T} f_{\alpha}\right) \geqslant a b>0$. Since $\mathcal{S}$ is a semigroup, we have $S T \in \mathcal{S}$, and so the proof is complete.

Corollary 3.4 gives the following.

Corollary 3.5. Let $\mathcal{S}$ be a multiplicative semigroup of order continuous positive operators on $L$. If $\phi_{\alpha}\left(S f_{\alpha}\right)=0$ for all $\alpha \in A$ and for all $S \in \mathcal{S}$, then for $\mathcal{S}$ the conclusions of Theorems 3.2 and 3.3 hold.

Proof. In this case $N_{S}=S$ for all $S \in \mathcal{S}$. Since $\mathcal{S}$ is a semigroup, we have $\phi_{\alpha}\left(S T f_{\alpha}\right)=$ 0 for all $\alpha \in A$ and for all $S, T \in \mathcal{S}$. Therefore, the hypothesis on the operators of Corollary 3.4 is satisfied. The assumption of Theorems 3.2 and 3.3 and Corollary 3.4 that $L$ is Dedekind complete is obviously used only to assure the decomposition from Proposition 1.6. Therefore, in view of the remark following Proposition 1.6, the space $L$ of Corollary 3.5 is not assumed to be Dedekind complete.

From Corollary 3.5 follows the most interesting result of this section.

Theorem 3.6. Let $\mathcal{S}$ be a multiplicative (respectively, an additive) semigroup of order continuous positive operators on an atomic normed Riesz space $L$. Assume that all of them are quasinilpotent at $f_{\alpha}$ for each $\alpha \in A$. Then there is a total ordering of the index set $A$ such that $\phi_{\beta}\left(N_{S} f_{\alpha}\right)=0$ whenever $S \in \mathcal{S}$ and $\alpha \leqslant \beta$. Furthermore, the chain $\mathcal{C}$ consists of $\mathcal{S}$-invariant bands, and it is maximal in the lattice of all bands of $L$. If, in addition, $L$ has order continuous norm, then the chain $\mathcal{C}$ is even maximal in the lattice of all closed subspaces of $L$.

Proof. As in the proof of Corollary 2.2 we show that $\phi_{\alpha}\left(S f_{\alpha}\right)=0$ for all $S \in \mathcal{S}$ and for all $\alpha \in A$. If $\mathcal{S}$ is a multiplicative semigroup, the result follows from Corollary 3.5 .

If $\mathcal{S}$ is an additive semigroup of positive operators, then it follows from Proposition 2.8 that every operator in the multiplicative semigroup generated by $\mathcal{S}$ is quasinilpotent at $f_{\alpha}$ for each $\alpha \in A$. Then apply what we have just proved.

Example 2.3 implies that the assumption of the last results that operators are order continuous can not be omitted. Also, if all members of $\mathcal{S}$ are order continuous and if the norm of $L$ is not order continuous, then $\mathcal{C}$ is not necessarily maximal in the lattice of all closed subspaces of $L$, as the following example shows.

Example 3.7. Let $S$ be the operator on $l^{\infty}$ defined by

$$
S\left(x_{1}, x_{2}, x_{3}, \ldots\right)=\left(x_{2}, x_{3} / 2, x_{4} / 4, x_{5} / 8, \ldots\right) .
$$

It is easily seen that $S$ is a quasinilpotent, compact, order continuous, positive operator. Let $\mathcal{S}$ be a semigroup generated by $S$. Then (uniquely) $A=\mathbb{N}, \mathcal{D}(A)=\mathbb{N} \cup\{\infty\}$ and $\mathcal{H}=\left\{H_{n}: 0 \leqslant n \leqslant \infty\right\}$, where $H_{n}=\{1,2,3, \ldots, n\}$. Since the closed ideal 
$\operatorname{cl}\left(\cup_{n=1}^{\infty} B_{H_{n}}\right)=c_{0}$ is not in $\mathcal{C}$, the chain $\mathcal{C}$ is not maximal in the lattice of all closed ideals in $l^{\infty}$, and hence in the lattice of all closed subspaces of $L$ as well.

As in [3] we conclude by mentioning that all the results in this paper remain true if in the definition of the local quasinilpotence we replace

$$
\lim _{n \rightarrow \infty}\left\|T^{n} f_{0}\right\|^{1 / n}=0 \text { by } \quad \liminf _{n \rightarrow \infty}\left\|T^{n} f_{0}\right\|^{1 / n}=0 .
$$

Acknowledgements. This work was supported by the Research Ministry of Slovenia. The author expresses his gratitude to Professor Matjaž Omladič for his helpful advice.

\section{References}

1. Y. A. Abramovich, C. D. Aliprantis and O. Burkinshaw, On the spectral radius of positive operators, Math. Z. 211 (1992), 593-607.

2. Y. A. Abramovich, C. D. Aliprantis and O. BURKINShaW, Invariant subspaces of operators on $l_{p}$-spaces, $J$. Funct. Analysis 115 (1993), 418-424.

3. Y. A. Abramovich, C. D. Aliprantis and O. Burkinshaw, Invariant subspace theorems for positive operators, J. Funct. Analysis 124 (1994), 95-111.

4. Y. A. Abramovich, C. D. Aliprantis and O. Burkinshaw, The invariant subspace problem: some recent advances, Rend. Istit. Mat. Univ. Trieste Suppl. 29 (1998), 1-76.

5. C. D. Aliprantis and O. Burkinshaw, Positive operators (Academic Press, Orlando, 1985).

6. G. BIRKhoff, Lattice theory, AMS Colloq. Publ., vol. 25 (Providence, RI, 1967).

7. M. D. Choi, E. A. Nordgren, H. Radjavi, P. Rosenthal and Y. Zhong, Triangularizing semigroups of quasinilpotent operators with non-negative entries, Indiana Univ. Math. J. 42 (1993), 15-25.

8. M. T. JAHANDIDEH, On the ideal-triangularizability of positive operators on Banach lattices, Proc. Am. Math. Soc. 125 (1997), 2661-1670.

9. M. T. JAHANDIDEH, Positive operators with $p$-hyperinvariant closed ideals. Preprint.

10. W. A. J. LuXemburg AND A. C. ZAAnen, Riesz spaces I (North-Holland, Amsterdam, 1971).

11. P. MEYER-NIEBERG, Banach lattices (Springer, 1991).

12. E. Nordgren, H. RadjaVi and P. Rosenthal, Triangularizing semigroups of compact operators, Indiana Univ. Math. J. 33 (1984), 271-275.

13. H. RADJAVI, On reducibility of semigroups of compact operators, Indiana Univ. Math. J. 39 (1990), 499-515.

14. J. R. RINGROSE, Compact non-self-adjoint operators (Van Nostrand Reinhold Math. Studies, London, 1971).

15. H. H. SCHAEFER, Banach lattices and positive operators, Grundlehren Math. Wiss. Bd. 215 (Springer, 1974).

16. H. H. SCHAEFER, Topologische Nilpotenz irreduzibler Operatoren, Math. Z. 117 (1970), 135-140.

17. A. C. ZAAnen, Riesz spaces II (North-Holland, Amsterdam, 1983). 